\title{
Correction to: Anterolateral ligament reconstruction improves the clinical and functional outcomes of anterior cruciate ligament reconstruction in athletes
}

\author{
Fawzy Hamido ${ }^{1,4}$ (D) Abdelrahman A. Habiba ${ }^{1}$ - Yousef Marwan ${ }^{1,2} \cdot$ Aymen S. I. Soliman $^{3} \cdot$ Tarek A. Elkhadrawe $^{3}$. \\ Mohamed G. Morsy ${ }^{3} \cdot$ Wael Shoaeb ${ }^{1} \cdot$ Ahmed Nagi $^{1}$
}

Published online: 20 August 2020

(c) European Society of Sports Traumatology, Knee Surgery, Arthroscopy (ESSKA) 2020

\section{Correction to: \\ Knee Surgery, Sports Traumatology, Arthroscopy \\ https://doi.org/10.1007/s00167-020-06119-w}

Authors would like correct the errors in Table 1 and typo in co-author name "Mohamed G. Morsy". The author name is corrected in author group and the corrected version of Table 1 updated below.

The original article can be found online at https://doi.org/10.1007/ s00167-020-06119-w.

Fawzy Hamido

fhf19633@yahoo.com

Abdelrahman A. Habiba

aakramh@yahoo.com

Yousef Marwan

yousefmarwan@hotmail.com

Aymen S. I. Soliman

Ssssoliman@hotmail.com

Tarek A. Elkhadrawe

elkhadrawe@yahoo.com

Mohamed G. Morsy

morsimoh@gmail.com

Wael Shoaeb

wael_shoaeb@yahoo.com
Ahmed Nagi

nagi_ot@hotmail.com

1 Division of Sports Medicine, Al-Razi Orthopaedic Hospital, Ministry of Health, Kuwait, Kuwait

2 Division of Orthopaedic Surgery, Department of Surgery, Faculty of Medicine, Health Sciences Center, Kuwait University, Kuwait, Kuwait

3 Department of Orthopaedic Surgery, Faculty of Medicine, Alexandria University, Alexandria, Egypt

4 Department of Orthopaedic Surgery, Al-Razi Orthopaedic Hospital, Safat, P.O. Box 24923, 13110 Kuwait, Kuwait 
Table 1 Background characteristics of the patients

\begin{tabular}{|c|c|c|c|c|}
\hline & Group A & & Group B & \\
\hline Characteristic & $52(100 \%)$ & & $50(100 \%)$ & \\
\hline Median age (range) years & (26) $18-40$ & & (24) 18-33 & \\
\hline Sex male/female no & $52 / 0$ & & $50 / 0$ & \\
\hline $\begin{array}{l}\text { Median duration between injury and opera- } \\
\text { tion (range) months }\end{array}$ & (3) $2.5-3.7$ & & (3) $2.5-3.8$ & \\
\hline Median follow-up time (range) & $60(55-65)$ months & & & \\
\hline Patients with Meniscal injury & $15(28.8 \%)$ & & $13(26.0 \%)$ & \\
\hline \multirow[t]{3}{*}{ Medial } & 10 & & 8 & \\
\hline & P.M. & M.S. & P.M. & M.S. \\
\hline & 8 & 2 & 7 & 1 \\
\hline \multirow[t]{3}{*}{ Lateral } & 6 & & 5 & \\
\hline & P.M. & M.S. & P.M. & M.S. \\
\hline & 4 & 2 & 4 & 1 \\
\hline \multirow[t]{3}{*}{ Both } & 1 & & 0 & \\
\hline & P.M. & M.S. & P.M. & M.S. \\
\hline & 2 & - & - & - \\
\hline Patients available for final assessment & 52 & & 50 & \\
\hline
\end{tabular}

Publisher's Note Springer Nature remains neutral with regard to jurisdictional claims in published maps and institutional affiliations. 